\title{
Dampened Mesolimbic Dopamine Function and Signaling by Saturated but not Monounsaturated Dietary Lipids
}

\author{
Cecile Hryhorczuk ${ }^{1,2}$, Marc Florea ${ }^{3}$, Demetra Rodaros', Isabelle Poirier ${ }^{4}$, Caroline Daneault ${ }^{5}$, \\ Christine Des Rosiers ${ }^{4,5}$, Andreas Arvanitogiannis ${ }^{3}$, Thierry Alquier ${ }^{1,6}$ and Stephanie Fulton ${ }^{*, 1,3,4}$ \\ 'Centre de Recherche du CHUM and Montreal Diabetes Research Center, Montreal, QC, Canada; ${ }^{2}$ Department of Physiology, Université de \\ Montréal, Montreal, QC, Canada; ${ }^{3}$ Center for Studies in Behavioral Neurobiology, Concordia University, Montreal, QC, Canada; ${ }^{4}$ Department of \\ Nutrition, Université de Montréal, Montreal, QC, Canada; ${ }^{5}$ Montreal Heart Institute, Montreal, QC, Canada; ${ }^{6}$ Department of Medicine, Université \\ de Montréal, Montreal, QC, Canada
}

\begin{abstract}
Overconsumption of dietary fat is increasingly linked with motivational and emotional impairments. Human and animal studies demonstrate associations between obesity and blunted reward function at the behavioral and neural level, but it is unclear to what degree such changes are a consequence of an obese state and whether they are contingent on dietary lipid class. We sought to determine the impact of prolonged ad libitum intake of diets rich in saturated or monounsaturated fat, separate from metabolic signals associated with increased adiposity, on dopamine (DA)-dependent behaviors and to identify pertinent signaling changes in the nucleus accumbens (NAc). Male rats fed a saturated (palm oil), but not an isocaloric monounsaturated (olive oil), high-fat diet exhibited decreased sensitivity to the rewarding (place preference) and locomotor-sensitizing effects of amphetamine as compared with low-fat diet controls. Blunted amphetamine action by saturated high-fat feeding was entirely independent of caloric intake, weight gain, and plasma levels of leptin, insulin, and glucose and was accompanied by biochemical and behavioral evidence of reduced DIR signaling in the NAc. Saturated high-fat feeding was also tied to protein markers of increased AMPA receptor-mediated plasticity and decreased DA transporter expression in the NAc but not to alterations in DA turnover and biosynthesis. Collectively, the results suggest that intake of saturated lipids can suppress DA signaling apart from increases in body weight and adiposity-related signals known to affect mesolimbic DA function, in part by diminishing DI receptor signaling, and that equivalent intake of monounsaturated dietary fat protects against such changes.

Neuropsychopharmacology (2016) 4I, 8II-82I; doi:I0.I038/npp.2015.207; published online 5 August 20I5
\end{abstract}

\section{INTRODUCTION}

Growing evidence suggests that dietary lipids can affect behavior and mental health. While well associated with obesity and cardiometabolic disease, excess intake of fat is increasingly linked to motivational and emotional impairments and the development of neuropsychiatric disorders (Bale et al, 2010; Hryhorczuk et al, 2013; Lai et al, 2014). Prolonged intake of high-fat food in rodents can reduce mesolimbic dopamine (DA) function and turnover (Davis et al, 2008), decrease nucleus accumbens (NAc) DA overflow (Geiger et al, 2009), modulate DA-related behaviors and striatal neuroadaptive responses (Sharma et al, 2013; Sharma and Fulton, 2013; Teegarden et al, 2009; Vucetic et al, 2012), and blunt DA reuptake in the NAc (Cone et al, 2013). We previously found that chronic high-fat feeding and weight gain in mice promotes depressive-like behavior, enhances physiological and behavioral responses to stress, and elicits

*Correspondence: Dr S Fulton, CRCHUM and Montreal Diabetes Research Center, 900 rue Saint-Denis, Office 8-428, Montreal, QC H2X 0A9, Canada, Tel: + I 5 I4 890 8000, ex 23602,

E-mail: stephanie.fulton@umontreal.ca

Received 13 March 2015; revised 2 June 2015; accepted 10 June 2015; accepted article preview online 14 July 2015 neuroplastic changes in the ventral tegmental area (VTA) and striatum coincident with reduced DA tone (Sharma et al, 2013; Sharma and Fulton, 2013).

Metabolic status has long been known to modulate the response to drugs of abuse (Carr, 2007). More recently it was established that peripherally derived hormones such as leptin and insulin, the levels of which are largely regulated by caloric load and diet composition, target the VTA to affect reward-relevant behaviors and psychostimulant action (Abizaid et al, 2006; Fulton et al, 2006; Labouebe et al, 2013). Though study of the interaction between metabolic hormones and DA-dependent behavior and signaling has received considerable attention, little is known about how dietary lipids affect mesolimbic function apart from major metabolic changes associated with weight gain.

Several lines of evidence suggest that peripheral metabolic tissues are differentially affected by saturated and unsaturated fat. Relative to diets comprising mostly unsaturated fat, a diet enriched in saturated fat can have deleterious metabolic actions that include increasing visceral fat mass (de Wit et al, 2012; Rosqvist et al, 2014). Here we studied the effects of prolonged intake of high-fat diets containing saturated or monounsaturated fat on DA-dependent behaviors and signaling in a manner that permitted assessment of 
dietary lipid impact separately from key metabolic changes known to affect mesolimbic function. Our results show that, independent of caloric intake, weight gain, and leptin, insulin, and glucose levels, saturated but not monounsaturated high-fat feeding decreases sensitivity to the rewarding effects of amphetamine (AMPH), suppresses the locomotorsensitizing effects of $A M P H$, attenuates D1 receptor-induced locomotor responses, and elicits neuroadaptations in the NAc coincident with decreased D1R signaling and enhanced AMPA receptor-mediated plasticity.

\section{MATERIALS AND METHODS}

\section{Animals and Diets}

All experimental manipulations were carried out in accordance with the guidelines of the CRCHUM Animal Care Committee and where applicable the Concordia University Animal Research Ethics Committee. Male Wistar rats (Charles River, St Constant, QC, Canada) weighing between 250 and $280 \mathrm{~g}$ upon arrival were housed 2 per cage in a reversed $12 \mathrm{~h}$ light/dark cycle (lights off at $1000 \mathrm{~h}$ ). Rats were assigned one of three customized diets: (1) a low-fat, control diet containing roughly equal amounts of monounsaturated and saturated fatty acids (FAs) ('CTL'; AIN-93G purified rodent diet with $17 \% \mathrm{Kcal}$ from fat derived from soybean oil, Dyets), (2) a monounsaturated high-fat diet (HFD; 'OLIVE', modified AIN-93G purified rodent diet with $50 \% \mathrm{Kcal}$ from fat derived from olive oil), or (3) a saturated HFD ('PALM', modified AIN-93G purified rodent diet with $50 \% \mathrm{Kcal}$ from fat derived from palm oil). As depicted in Supplementary Table S1, the three diets were designed for equal sucrose content, and the two HFDs were matched for protein, fat content, and caloric density. Rats were singly housed for feeding and body weight measures. Food intake and body weight were measured once per week just before dark cycle onset. All behavioral tests were conducted 8 to 9 weeks after the start of the diet and animals were maintained on their respective diet throughout each experiment.

\section{Drugs}

d-Amphetamine sulfate (AMPH; Sigma-Aldrich, Dorset, $\mathrm{UK}$ ) was dissolved in $0.9 \%$ saline (SAL) and administered intraperitoneally (IP) at $0.75,1.5,3$, or $4.5 \mathrm{mg} / \mathrm{kg}$ in SAL. The D1 receptor agonist SKF82958 (Santa Cruz, Dallas, TX) was dissolved in $0.9 \%$ SAL and administered IP at $0.1 \mathrm{mg} / \mathrm{kg}$.

\section{Amphetamine-Induced Conditioned Place Preference}

Rats $(n=75)$ from each diet group were randomly assigned to one of the three AMPH doses. Conditioned place preference (CPP) chambers had two compartments distinguished by tactile and visual cues and a neutral middle compartment connected by manual guillotine doors (Med Associates, St Albans, VT). All testing was performed during the dark phase.

Pretest. On the first day of the experiment, rats were placed in the middle compartment and allowed to explore the entire apparatus for $15 \mathrm{~min}$. Time spent in each compartment was recorded (MedPC-IV Software, Med Associates). Assignment of AMPH treatment to a compartment (AMPH-paired) and to the initial preferred or nonpreferred side was counterbalanced within each diet group.

Conditioning (3 days). Rats were injected with SAL and confined to one side of the apparatus for $30 \mathrm{~min}$. After $4 \mathrm{~h}$, they were injected with AMPH $(1.5,3.0$, or $4.5 \mathrm{mg} / \mathrm{kg})$ and confined to the other side of the apparatus.

Posttest. Rats were placed in the middle compartment with the doors open and were free to explore the entire apparatus for $15 \mathrm{~min}$. Time spent in each compartment was recorded and compared with pretest measures. Results are expressed as time spent in the AMPH-paired side minus time spent in the SAL-paired side during pre- and post-test days.

\section{Amphetamine Sensitization}

Testing was carried out in chambers $(42 \times 42 \times 21 \mathrm{~cm})$ using the Versamax Animal Activity Monitoring system (AccuScan Instruments, Columbus, OH, USA).

Habituation. Following 8 weeks on one of three diets, a new cohort of rats $(n=46)$ was placed in the chambers and locomotor activity was recorded for $6 \mathrm{~h}$ in the middle of the dark phase to assess basal locomotion.

Pretreatment. The following day, rats from each diet group were split in two groups that were matched for average body weight. Rats were injected with AMPH $(1.5 \mathrm{mg} / \mathrm{kg})$ or SAL once every 2 days for 10 days (5 injections). Rats were kept in the test chambers during the entire pretreatment period and returned to their home cage after the last injection.

Sensitization. At 7 days after the last pretreatment injection, all rats received $0.75 \mathrm{mg} / \mathrm{kg}$ of AMPH. Locomotor activity was recorded $1 \mathrm{~h}$ before and $2 \mathrm{~h}$ after injection.

\section{D1 Receptor-Induced Locomotion}

A separate group of rats $(n=25)$ were used to test the locomotor response to a D1R agonist (SKF82958). A $0.1 \mathrm{mg} / \mathrm{kg}$ dose was chosen as it was shown to be the lowest dose to induce locomotion in Wistar rats (Wang and McGinty, 1997). Rats were acclimated to locomotor chambers described above 1 day before testing. Locomotor activity was measured $1 \mathrm{~h}$ before and $3 \mathrm{~h}$ after injection in the middle of the light phase.

\section{Plasma Biochemical Analyses}

Rat $(n=49)$ were killed after 8 weeks of diet. Blood was collected in EDTA-coated tubes and centrifuged at $11000 \mathrm{~g}$ for $15 \mathrm{~min}$ at $4{ }^{\circ} \mathrm{C}$. Plasma was kept at $-20^{\circ} \mathrm{C}$ until use. Leptin and insulin levels were analyzed by ELISA kits (EMD Millipore, Billerica, MA and ALPCO Diagnostics, Salem, $\mathrm{NH})$. Glycemia was assessed from tail blood after 7.5 weeks 
on each diet using a glucometer (Accu-Check, Roche Diagnostics, Basel, Switzerland).

\section{Gas Chromatography-Mass Spectrometry}

Plasma fatty acid composition was measured by gas chromatography-mass spectrometry (GC-MS) using a previously described modified method (Gelinas et al, 2011). Briefly, total lipids were extracted with a mixture of methyltert-butyl ether (MTBE), methanol, and water, according to Matyash et al (2008) and FAs were analyzed as their methyl ester (FAME) derivatives. Injections $(2 \mu \mathrm{l})$ were performed into an Agilent $6890 \mathrm{~N}$ gas chromatograph equipped with a Select FAME CP7420 capillary column (100 m; $250 \mu \mathrm{m}$ inner diameter; $230 \mu \mathrm{m}$ thickness) coupled with a 5975 Mass Selective Detector operated in PCI mode using ammonia as reagent gas. FAs were identified according to their retention time and $m / z$, and their concentration was calculated according to a mix of internal and external labeled standards with known concentrations.

\section{High-Performance Liquid Chromatography}

At 10 days after the CPP task, rats (from the $3.0 \mathrm{mg} / \mathrm{kg}$ AMPH condition) were anesthetized, decapitated, and brains removed and frozen. Brains were stored at $-80{ }^{\circ} \mathrm{C}$ until further use. Brain punches of the NAc were collected on $300 \mu \mathrm{m}$ brain sections and kept at $-80^{\circ} \mathrm{C}$. At 1 week before the experiment, $100 \mu \mathrm{l}$ of filtered $0.1 \mathrm{M}$ phosphate buffer was added to the punches. Tubes were vortexed for $20 \mathrm{~s}$ and samples were centrifuged at 10000 r.p.m. for $10 \mathrm{~min}$ at $4{ }^{\circ} \mathrm{C}$. Then, $150 \mu \mathrm{l}$ of $0.1 \mathrm{M} \mathrm{NaOH}$ was added to the protein pellets and concentrations were determined using the BCA Protein Assay Kit (Thermo Fisher Scientific, Rockford, IL). The supernatant was filtered $(0.45 \mu \mathrm{m}$ syringe-driven filter units, EMD Millipore) and $10 \mu \mathrm{l}$ was used to determine DA, homovanillic acid (HVA), and 3,4-Dihydroxyphenylacetic acid (DOPAC) content as previously described (Hernandez et al, 2007).

\section{Western Immunoblotting}

Brains from a separate group of drug-naive rats $(n=36)$ after 8 weeks of diet were rapidly removed and frozen. Tissue punches of the NAc (core and shell) were homogenized in $50 \mu \mathrm{l}$ of lysis buffer-20 mM Tris, pH 7.5; $150 \mathrm{mM} \mathrm{NaCl}$; $1 \mathrm{mM} \mathrm{Na} 2$ EDTA; $1 \mathrm{mM}$ EGTA; $1 \%$ Triton; $2.5 \mathrm{mM}$ sodium pyrophosphate; $1 \mathrm{mM} \beta$-glycerophosphate; $1 \mathrm{mM}$ sodium orthovanadate; $1 \mu \mathrm{g} / \mathrm{ml}$ leupeptin; $1 \mathrm{mM}$ phenylmethylsulfonyl fluoride; $1 \mu \mathrm{l}$ phosphatase inhibitors cocktails 2 , and $1 \mu \mathrm{l}$ phosphatase inhibitors cocktails 3 (Sigma-Aldrich). Homogenates were centrifuged at $13000 \mathrm{~g}$ for $10 \mathrm{~min}$ at $4^{\circ} \mathrm{C}$. Supernatants were collected and proteins quantified with a BCA Protein Assay Kit (Thermo Fisher Scientific). Proteins were separated on a $10 \%$ sodium dodecyl sulfatepolyacrylamide gel by electrophoresis and transferred onto a nitrocellulose membrane (Bio-Rad, Bedford, MA). Membranes were incubated for $2 \mathrm{~h}$ at room temperature in $5 \%$ skim milk-Tris-buffered saline-0.1\% Tween-20 (TBS-T) (5\% SKM) to block nonspecific binding sites and probed overnight at $4{ }^{\circ} \mathrm{C}$ with primary antibody (anti-TH, $1: 40000$, Millipore MAB318; anti-DAT, 1:1000, Millipore AB2231;
anti-D1R, 1:500, Millipore AB1784P; anti-phospho DARPP-32 ${ }^{\text {Thr34 }}, 1: 1000$, Santa Cruz sc135687; antiphospho DARPP-32 ${ }^{\text {Thr75 }}$, 1:1000, Cell Signaling 2301s; anti-DARPP-32, $1: 1000$, Cell Signaling 2302; anti-GAPDH, $1: 50$ 000, Cell Signaling 2118; anti-GLUR1, $1: 1000$, Santa Cruz sc13152) in 5\% SKM except for D2R (1:1000; Millipore AB5084P) and phospho-GluR1 Ser845 (1:1000; Santa Cruz sc135699) that were incubated in 3\% BSA (Sigma-Aldrich). Following rinses in TBS-T, membranes were incubated for $2 \mathrm{~h}$ at room temperature with the appropriate secondary antibody coupled to horseradish peroxidase (Goat antiRabbit IgG-HRP, $1: 10$ 000, Bio-Rad 170-6515 or Goat antiMouse IgG-HRP, 1:10 000, Bio-Rad 170-6516) in 5\% SKM. Antibodies were revealed by chemiluminescence using the Western Lightning Plus-ECL Kit (Perkin Elmer, Waltham, MA) and autoradiography films (Harvard Apparatus Canada). Signals were scanned and analyzed by densitometry using ImageJ (http://rsbweb.nih.gov/ij/). Protein size was determined using a protein standard (Bio-Rad).

\section{Statistical Analyses}

Results were analyzed using GraphPad Prism v6. A one-way analysis of variance (ANOVA) with Bonferroni post-tests was used to analyze AMPH locomotor sensitization, metabolic, and protein expression data. Analyzed and illustrated AMPH locomotor sensitization results represent AUC values (locomotor activity $2 \mathrm{~h}$ after injection) of AMPH-treated rats normalized to AUC values of saline-treated rats. A linear regression analysis with dose as a continuous variable was employed to determine the effects of diet on AMPH CPP across the three doses tested. At each AMPH dose, one-tailed $t$-tests were used to assess AMPH place preference within each diet. For analysis of the D1 receptor agonist data, we used a nonparametric Kruskal-Wallis test with post hoc Dunn's pairwise comparisons because of the detection of unequal variances between diet groups. Data are presented as mean \pm SEM. Statistical significance was set at $p \leqslant 0.05$.

\section{RESULTS}

\section{Diet-Induced Metabolic Outcomes}

All animals gained weight over the course of the diet manipulation; however, there were no differences in caloric intake and body weight gain between the CTL, OLIVE, and PALM groups after 8 weeks (Figure $1 \mathrm{a}$ and $\mathrm{b}$ ). Palmitate and oleate are the two most abundant circulating long-chain fatty acids and are major constituents of the PALM and OLIVE diets, respectively (Supplementary Table S1). To verify that dietary interventions modified the plasma composition of these FAs in the expected manner, we measured plasma FA levels by GC-MS. As shown in Figure 1c, the plasma ratio of oleate to palmitate largely matches the diet ratio of these FAs (Figure 1c, inset). Finally, we measured the circulating concentrations of three metabolic signals known to affect mesolimbic DA tone, namely glucose, leptin, and insulin, and found no differences between the PALM, OLIVE, and CTL groups following the dietary intervention (Figure 1d-f). 
a

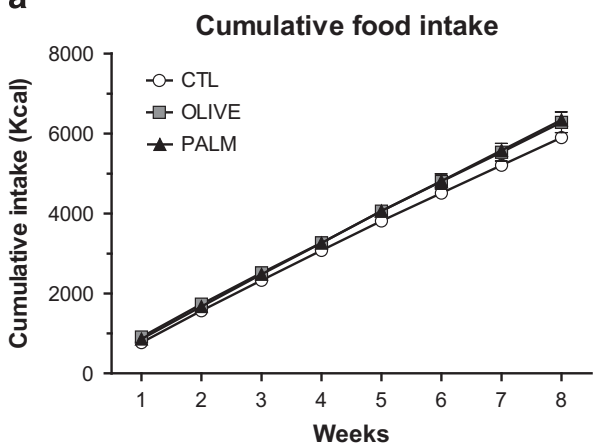

C

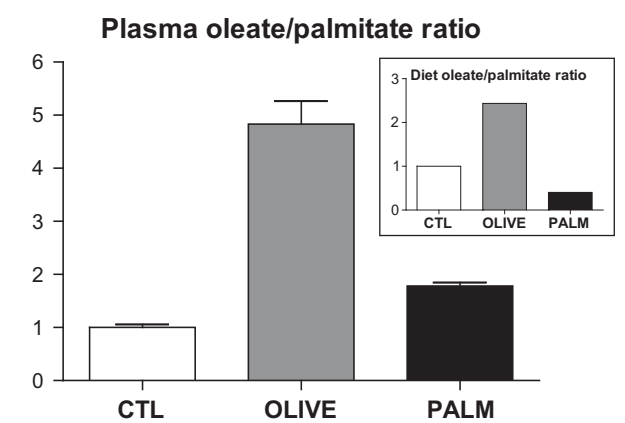

e

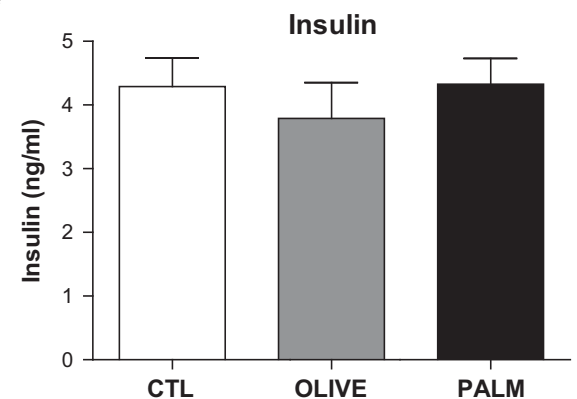

b

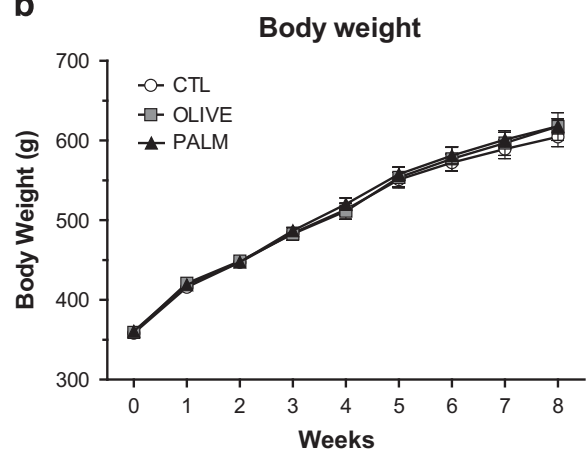

d

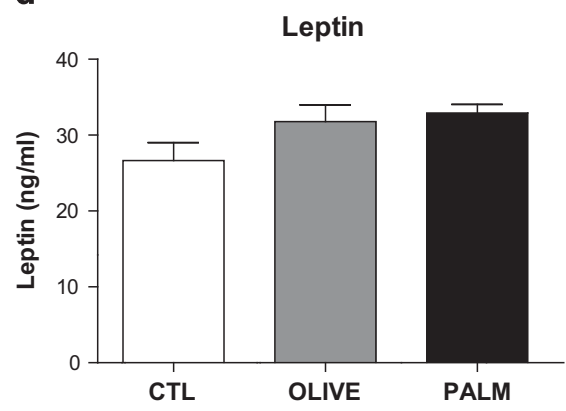

f

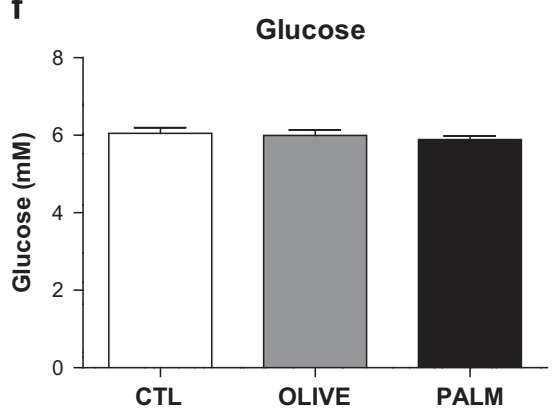

Figure I Similar food intake and weight gain across CTL, PALM, and OLIVE diet groups. (a) Cumulative food intake and (b) body weight were assessed weekly during 8 weeks ( $n=11-12$ per group). (c) The ratio of oleate/palmitate in plasma ( $n=7-8$ per group) reflected that found in the two high-fat diets (insert). Plasma levels of ( $d$ leptin ( $n=9$ per group) and (e) insulin ( $n=8$ per group) and ( $f$ fasting blood glucose $(n=16-18$ per group) were similar between groups after 8 weeks on respective diets. Mean \pm SEM.

\section{A Saturated HFD Decreases Sensitivity to the Rewarding Effects of AMPH}

To determine whether prolonged intake of a saturated or a monounsaturated HFD can affect mesolimbic DA reward function, we studied the effects of diet on AMPH place preference using three doses of AMPH, each dose administered to a separate group of rats. At the lowest dose tested $(1.5 \mathrm{mg} / \mathrm{kg})$, only the CTL group developed a significant AMPH place preference, whereas the OLIVE group showed a modest, nonsignificant, place preference, and the PALM group failed to show preference for the AMPH-paired side (Figure 2a). Both the CTL and OLIVE groups but not the PALM group demonstrated a significant CPP at a higher AMPH dose $(3.0 \mathrm{mg} / \mathrm{kg}$, Figure $2 \mathrm{~b})$. Finally, at the highest dose tested $(4.5 \mathrm{mg} / \mathrm{kg})$, rats fed the OLIVE diet exhibited place preference and there was a near-significant CPP in rats fed the PALM diet (Figure 2c). As illustrated in Figure 2d, the magnitude of AMPH CPP for the CTL and OLIVE groups was similar across the three doses whereas preference for the AMPH-paired side increased as a function of dose for the PALM group (linear regression, $p \leqslant 0.05$ vs CTL group).

\section{Saturated High-Fat Feeding Suppresses the Locomotor-Sensitizing Effects of $\mathrm{AMPH}$}

To further assess mesolimbic function we examined the locomotor-sensitizing effects of AMPH following the three diets. At 7 days following AMPH or SAL pretreatment injections, locomotor activity was measured in response to a lower dose of AMPH according to a protocol of repeated intermittent AMPH exposure (Robinson and Becker, 1986) (Figure 3a). Whereas there was no difference in pretreatment (Figure $3 \mathrm{~b}$ ) or in basal locomotor activity (Figure 3c, inset; measured for $6 \mathrm{~h}$ before treatments), the locomotorsensitizing effects of AMPH were attenuated in the PALM group relative to CTL (Figure $3 c$ and $d$ ). 
a

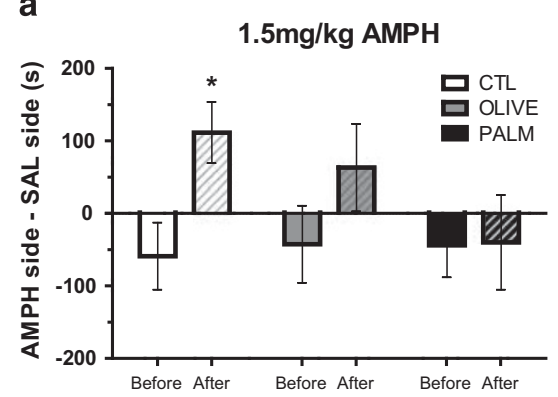

C

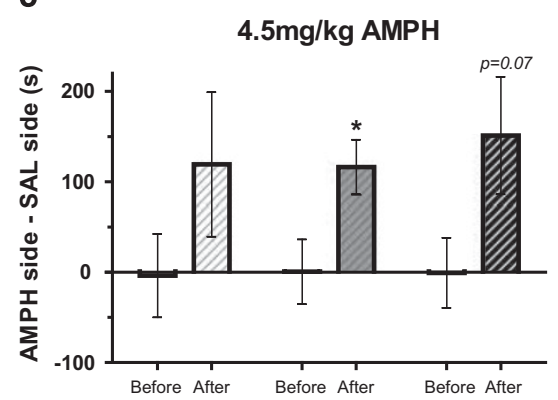

b

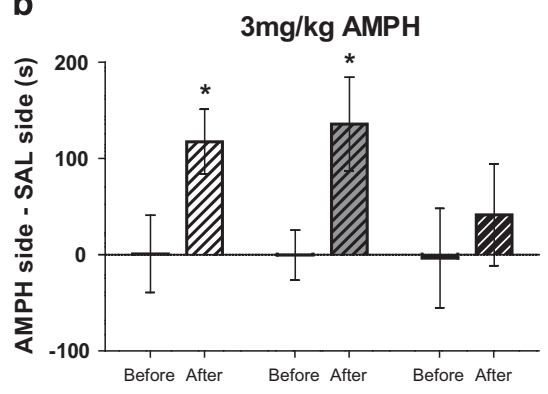

d

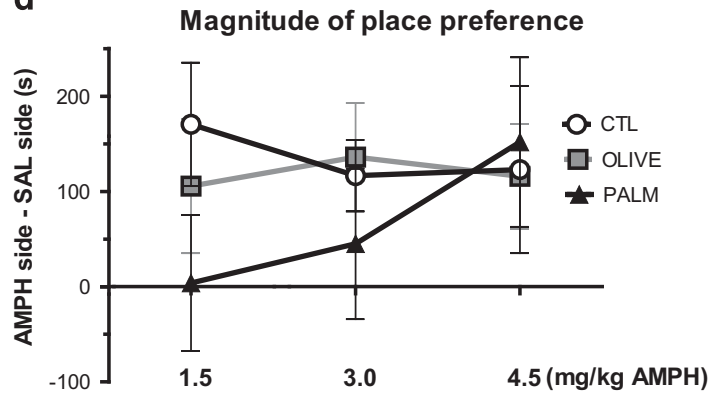

Figure 2 Amphetamine-induced conditioned place preference is suppressed by saturated high-fat feeding. Results are expressed as time spent in the AMPH-paired side minus time spent in the SAL-paired side before and after conditioning. (a) Only rats fed the CTL diet acquired place preference for $1.5 \mathrm{mg} / \mathrm{kg}$ AMPH ( $n=10-1$ I per group). (b) At the dose of $3 \mathrm{mg} / \mathrm{kg}$ AMPH, CTL and OLIVE rats acquired place preference but not the PALM rats ( $n=7-8$ per group). (c) The $4.5 \mathrm{mg} / \mathrm{kg}$ AMPH dose elicited a place preference in the OLIVE group and a near-significant effect in the PALM group ( $p=0.069 ; n=7-8$ per group). (d) Magnitude of place preference for each diet group across the three doses of AMPH used. A multiple regression analysis across the dose range used revealed differences between PALM and the CTL group. Solid bars: before conditioning; hatched bars: after conditioning. SAL: saline; AMPH: amphetamine. Mean \pm SEM; $* p \leq 0.05$, after vs before conditioning.

\section{Saturated High-Fat Feeding Alters Expression of DA-Related Proteins without Affecting DA Turnover in the NAc}

We next sought to determine whether the observed behavioral changes elicited by saturated high-fat feeding are associated with alterations in DA-related protein expression and DA turnover in the NAc. As psychostimulant-induced locomotion requires NAc AMPA receptor activation (Wolf, 1998), we also measured the expression of the phosphorylated GluR1 subunit of the AMPA receptor. As shown in Figure 4a, dopamine transporter (DAT) protein levels, both mature glycosylated (membrane bound, $75 \mathrm{kDa})$ and immature nonglycosylated $(50 \mathrm{kDa})$ forms, were decreased in the NAc of the PALM group relative to CTLs. On the other hand, levels of D1R, pDARPP- $32^{\text {Thr75 }}$, and pGluR1 ${ }^{\text {Ser845 }}$ expression were increased in the PALM group as compared with CTLs. Expression of TH, D2R, and pDARPP-32 ${ }^{\text {Thr34 }}$ did not differ between diet groups. Consistent with our behavioral results showing that the monounsaturated HFD largely fails to alter mesolimbic DA function, we did not find changes in any of the measured proteins in the OLIVE group. As the biochemical and behavioral alterations could be due to changes in DA metabolism, we quantified DA turnover in NAc samples via HPLC. NAc DA turnover, expressed as the ratio between DOPAC and DA, did not differ between groups (Figure $4 \mathrm{~b}-\mathrm{d}$ ).

\section{Saturated High-Fat Feeding Attenuates the Locomotor Response to a D1R Agonist}

As an inhibitor of cAMP-dependent protein kinase (PKA), the observed increases in pDARPP-32 at Thr75 are suggestive of decreased D1R-PKA signaling. Given that D1R and its downstream effector PKA are critical for the sensitizing (Vezina, 2004), conditioned activity (Gerdjikov et al, 2007), and conditioned rewarding effects of AMPH (Beninger et al, 2003), we investigated locomotor activity following administration of a D1R agonist. Relative to the saline-injected control condition (Figure 5a), the agonist effectively stimulated locomotor activity in each diet group (Figure 5b); however, the locomotor response to the D1 agonist was reduced in rats fed the PALM diet relative to the OLIVE group.

\section{DISCUSSION}

Beyond its adverse effects on metabolic and cardiovascular health, emerging findings are linking excess dietary fat and the development of obesity to impaired neural signaling and neurological disorders such as depression (reviewed in Hryhorczuk et al, 2013 and Martinez-Gonzalez and Martin-Calvo, 2013). Comparing two principal lipid classes, here we find that chronic intake of a saturated HFD independent of obesity or weight gain suppressed DA-dependent behaviors, whereas an isocaloric diet consisting of monounsaturated lipids was protective. This dampening of mesolimbic function by saturated fat intake is tied to attenuated D1R signaling, lowered DAT expression, and increased AMPA receptor-mediated plasticity in the NAc. Of significance, the observed changes in mesolimbic DA function did not rely on changes in key adiposity hormones known to modulate DA neurotransmission and thereby 
a

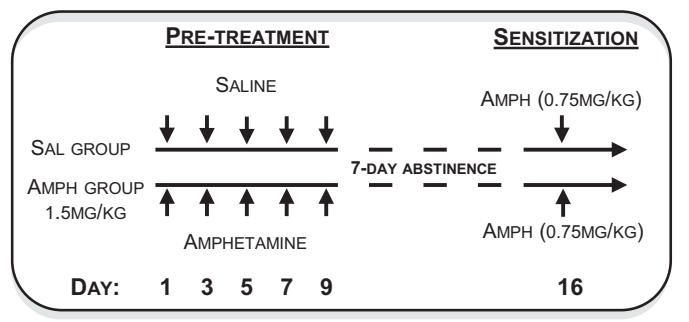

C

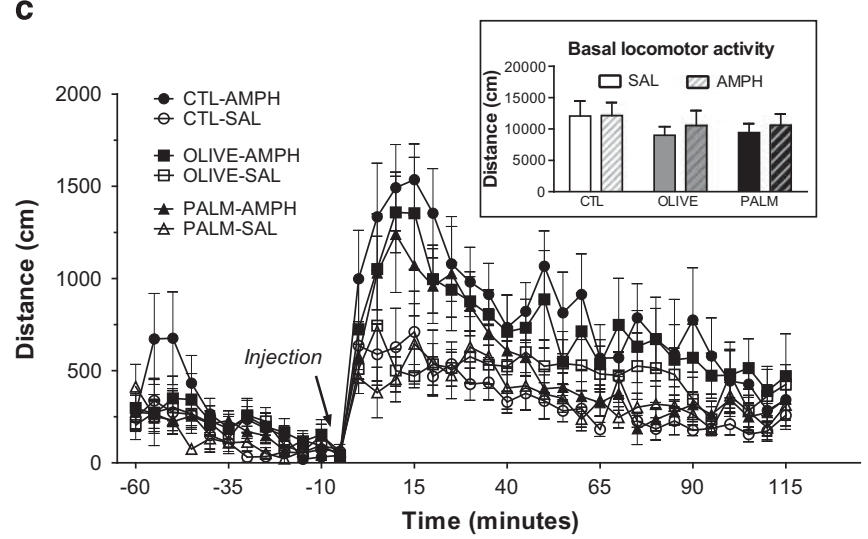

b

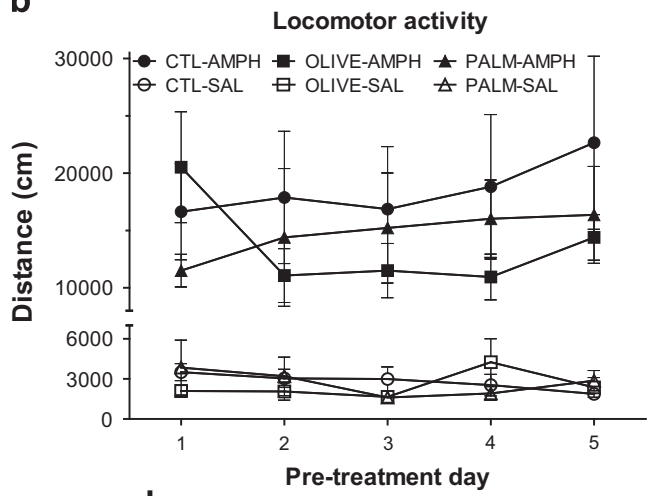

d

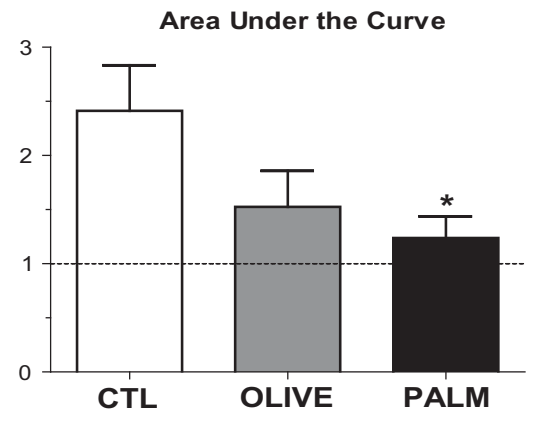

Figure 3 Dampened amphetamine locomotor sensitization in rats fed the saturated high-fat diet. (a) AMPH sensitization experimental layout. (b) AMPH increased locomotor activity compared with SAL-injected animals throughout the pretreatment phase $(n=7-9$ per group). (c) AMPH ( $1.5 \mathrm{mg} / \mathrm{kg}$ ) pretreatment increased response to the locomotor activating effect of a single $0.75 \mathrm{mg} / \mathrm{kg}$ AMPH injection. Basal locomotor activity was similar in all groups (insert) ( $n=7-9$ per group). (d) AMPH locomotor sensitization was reduced in rats fed the PALM diet as compared with controls. Relative AUC: the response of AMPH-treated rats is expressed in fold change relative to that of SAL-treated counterparts $(n=8-9$ per group). SAL: saline; AMPH: amphetamine. Mean \pm SEM; * $\leq 0.05$ vs CTL group.

suggest that saturated dietary lipids can diminish rewardrelevant function apart from neuroadaptive processes triggered by weight gain and obesity.

The dietary interventions aimed to assess the influence of fat type and amount on AMPH-induced behaviors and DA signaling. In order to remove the potential contribution of nutritional components other than fat, diets were designed to be ingredient matched, including sources of carbohydrate and protein, and to have equal sucrose content. Although the type and amount of dietary fat, namely saturated and unsaturated, can lead to different metabolic outcomes in both rodents (Buettner et al, 2006; Carsia et al, 2008; Keita et al, 2013) and humans (Kien et al, 2014; Rosqvist et al, 2014), caloric intake and body weight were similar across diet groups after 8 weeks in the present study, an effect that was reproducible over multiple cohorts we studied. Moreover, glucose, leptin, and insulin levels were comparable across groups at the end of the 8-week regimen. Extending the period of high-fat feeding would likely result in significant metabolic changes as it is seen with rats of same strain fed HFD for longer periods of time (Hoefel et al, 2011; Panchal et al, 2011; Sampey et al, 2011). We cannot rule out that our saturated HFD regimen diminished central sensitivity to leptin and insulin or that alterations in other hormones contribute to our results. However, it should be noted that HFD-induced central leptin and insulin resistance is characteristically associated with elevated plasma concentrations of these hormones and increased body weight (Munzberg et al, 2004; Wang et al, 2001b).

Previous studies report that diet-induced obesity (DIO) (Davis et al, 2008; Morales et al, 2012) or genetic susceptibility to DIO (Thanos et al, 2010) impairs acquisition of place preference induced by psychostimulant drugs. Here our results suggest that this effect is contingent on lipid class but not on weight gain and associated metabolic changes. Furthermore, we extend these suppressive effects of saturated high-fat feeding to AMPH psychomotor sensitization, D1Rmediated locomotor responses, and distinct neuroadaptive changes in the NAc. Prolonged intake of the saturated HFD (PALM) reduced the effectiveness of AMPH to elicit a place preference, an effect not observed with the monounsaturated (OLIVE) HFD. The saturated HFD prevented AMPH place preference acquisition at the lowest dose tested but became apparent as AMPH dose escalated to suggest that saturated high-fat feeding lowers the sensitivity to the conditioned rewarding effects of AMPH. Saturated high-fat feeding is reported to disrupt the blood-brain barrier at the level of the hippocampus and impair learning and memory (Kanoski et al, 2010), advancing the possibility that reduced sensitivity of the PALM group in the CPP task could be linked to such impairments. However, hippocampal dysfunction appears to be tied to weight gain and increased adiposity not observed 
a

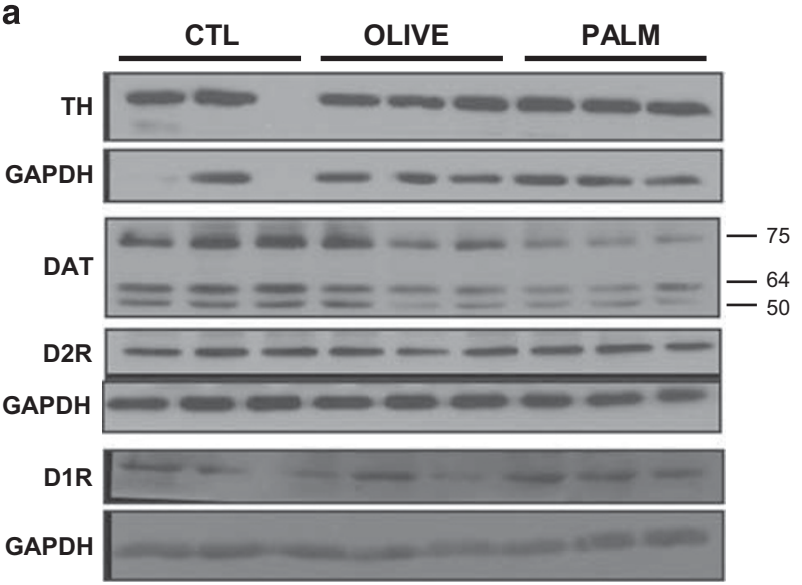

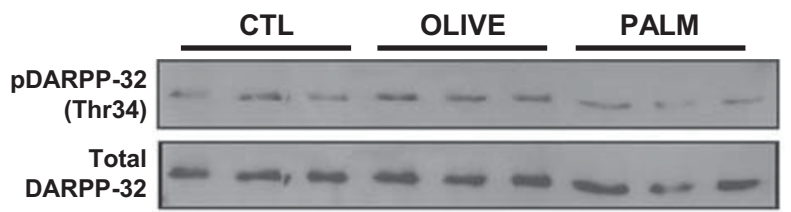
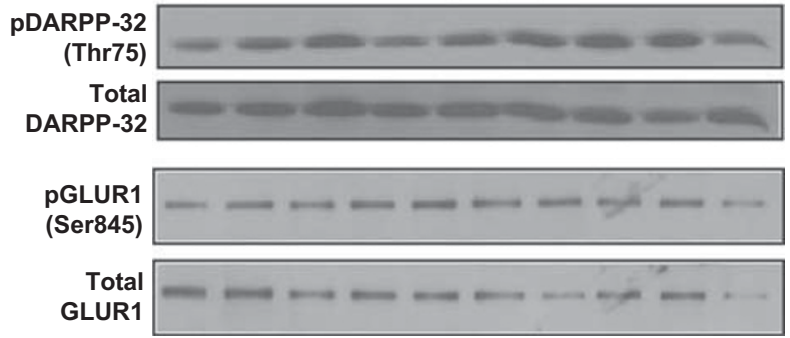

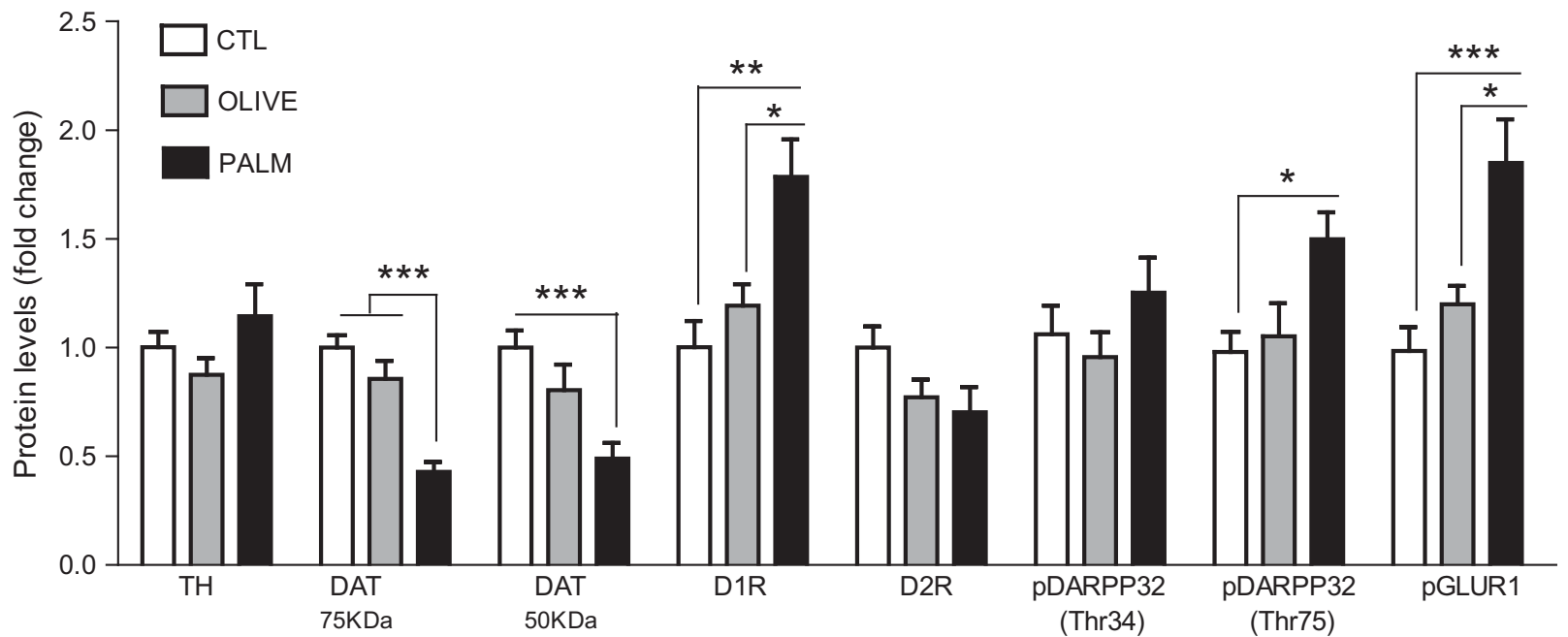

b

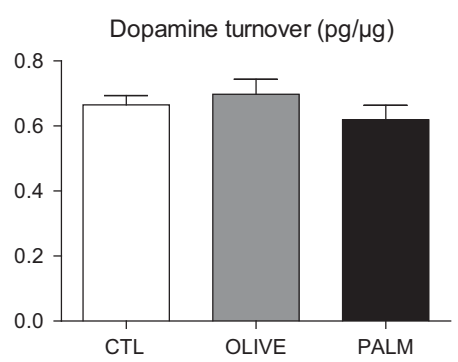

C

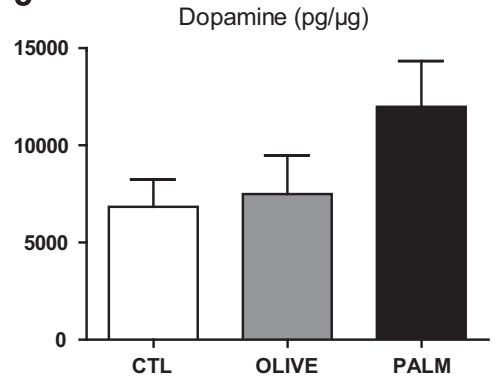

d

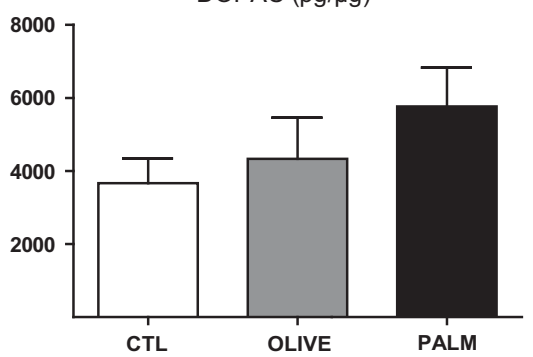

Figure 4 Effects of high-fat diets on protein expression and dopamine turnover in the nucleus accumbens. (a) The PALM diet caused a decrease in DAT levels, whereas PGLURI, DIR, and pDARPP-32 ${ }^{\text {hr75 }}$ were increased $(n=7-13$ per group). (b-d) There were no changes in (b) dopamine turnover, (c) dopamine, and (d) DOPAC content in the nucleus accumbens ( $n=6-8$ per group). Dopamine turnover is expressed as the ratio between the dopamine metabolite DOPAC and dopamine. DOPAC: 3,4-dihydroxyphenylacetic acid. Mean \pm SEM; $* p \leq 0.05, * * p \leq 0.01, * * * * 1 \leq 0.001$.

in the present study (Davidson et al, 2012). Our observations are in line with those of Davis et al (2008) showing that highfat feeding without added weight gain diminishes AMPH place preference. In this study, however, access to the HFD was restricted (pair-feeding) contrary to the free-feeding conditions used here. Consistent with the CPP results, we found that the saturated HFD suppressed AMPH-induced locomotor sensitization. Although not statistically significant, animals consuming the monounsaturated HFD showed slightly reduced AMPH locomotor sensitization and appeared less responsive to the conditioned rewarding effects of AMPH at the lowest dose tested. Thus, we cannot exclude the possibility that consumption of the OLIVE HFD over a longer time period may also blunt mesolimbic signaling. Of note, given that locomotor responses to AMPH were similar across diet groups during the pretreatment phase of the locomotor sensitization test, it is unlikely that the effects of the PALM diet on AMPH-induced behavioral responses is because of changes in the pharmacokinetic properties of the drug. Rather, saturated high-fat feeding appears to selectively 
a

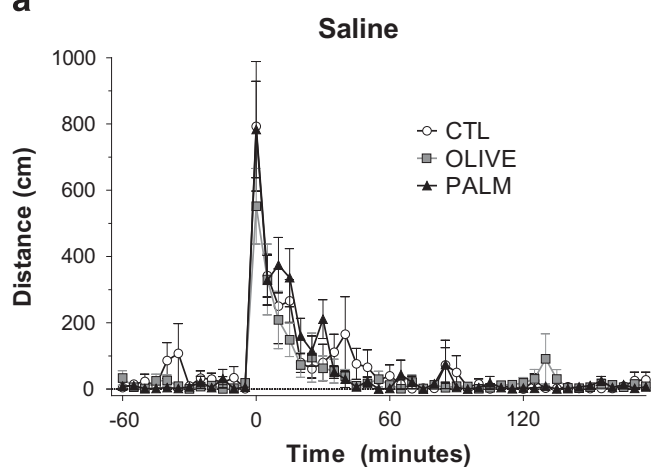

b

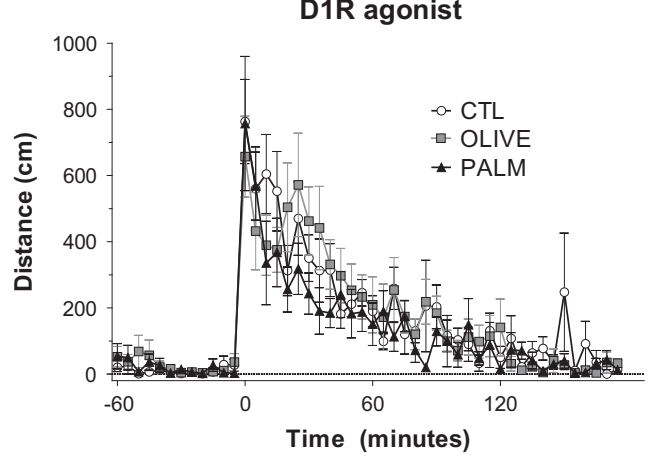

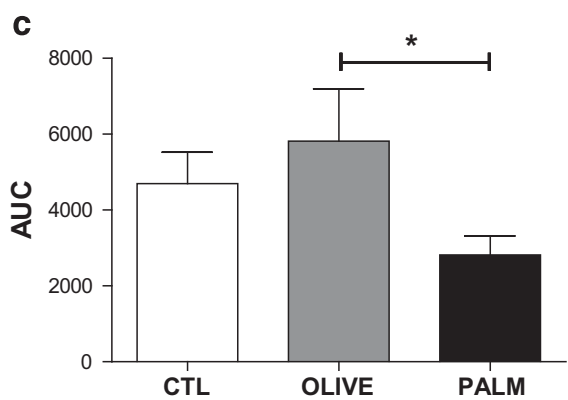

Figure 5 Locomotor activity in response to a DIR agonist is attenuated by saturated HFD relative to the monounsaturated HFD. Locomotor activity was measured as total $X-Y$ distance after injection of (a) saline and (b) the DIR agonist SKF82958 (0.I mg/kg) ( $n=8-9$ per group). (c) Area under the curve $(A \cup C)$ values $=\mathrm{AUC}_{\text {agonist }}-\mathrm{AUC}_{\text {saline. }}$ Mean $\pm \mathrm{SEM} ; *$ $\leq 0.05$

modulate signaling mechanisms related to the neuroplastic changes associated with repeated AMPH treatment as opposed to those responsible for its primary neurophysiological effects.

Given that chronic food restriction increases the reward value of drugs of abuse (Carr, 2007), it is not surprising that sustained caloric overload has inverse actions. Consistent with this observation, heightened reward responses during food restriction have been linked to decreased leptin (Fulton et al, 2000) and insulin signaling (Carr et al, 2000). However, our findings suggest that increased caloric intake, body mass, leptin, insulin, and glucose are not necessary for reduced mesolimbic DA function by high-fat feeding. One possibility is that excess dietary saturated fat results in higher circulating levels of saturated long-chain fatty acids like palmitate that may foster greater central actions of these FAs. Indeed, oleate and palmitate have distinct intracellular metabolism in the brain (Taib et al, 2013) and palmitate has been shown to act centrally to impair signaling processes including those that lead to reduced leptin receptor signaling (Benoit et al, 2009; Kleinridders et al, 2009). In line with this prospect, we found that the ratio of oleate to palmitate in plasma matched that of the different diets. Another possibility is that these dietary lipids differentially modulate gut ethanolamines that have been shown to influence DA tone via a gut-brain axis (Tellez et al, 2013). A final explanation stems from evidence that overconsumption of saturated fats stimulate peripheral and central immune responses (Maric et al, 2014; Thaler et al, 2012; Valdearcos et al, 2014; van Dijk et al, 2009). However, it should be noted that such proinflammatory processes have been consistently tied to a significant increase in body weight.
Behavioral perturbations by saturated HFD were accompanied by modifications in the expression of several DArelated proteins in the NAc. Levels of DAT were lower in the NAc of rats fed the saturated HFD. This finding is in agreement with a report by Cone et al (2013) showing a reduction in the expression of the plasma membraneassociated immature form of DAT in the NAc of nonobese rats exposed to 6 weeks of HFD that was associated with reduced DA reuptake. Decreased DAT expression by saturated lipids may well contribute to diminished AMPH action as AMPH blocks DA reuptake and elicits reverse DA transport through DAT (Jones et al, 1998; Sulzer et al, 1995). Indeed, DAT expression correlates positively with AMPH potency (Siciliano et al, 2014) and AMPH fails to evoke DA release and locomotion in mice lacking DAT (Cagniard et al, 2014; Jones et al, 1998; Spielewoy et al, 2001).

In addition, our data suggest that reduced D1R signaling could be a factor underlying blunted AMPH action. Although the saturated HFD increased D1R expression in the NAc, this could be compensatory to decreased D1R signaling given that saturated HFD increased NAc pDARPP-32 ${ }^{\text {Thr75 }}$, a marker of reduced D1R-PKA signaling, and attenuated locomotor responses to a D1R agonist relative to rats fed the monounsaturated HFD. Indeed, D1R-PKA signaling plays a critical role in the expression of AMPH place preference and sensitization (Hiroi and White, 1991; Vezina, 2004), and thus saturated HFD may diminish AMPH conditioned responses via modifications in D1R. Interestingly, phosphorylation of DARPP $-32^{\text {Thr75 }}$ is under the control of cyclin-dependent kinase 5 (CDK5) and its activation by the transcription factor $\Delta$ FosB, the two molecules that are upregulated in the NAc by chronic HFD (Sharma 
and Fulton, 2013; Teegarden et al, 2009). Contrary to reports that D2 receptor binding is suppressed in obese individuals (Stice et al, 2008; Volkow et al, 2008; Wang et al, 2001a) and D2 receptor expression is reduced in diet-induced (Johnson and Kenny, 2010) or genetically induced (Hamdi et al, 1992) obese rodent models, we did not find significant decreases in D2 receptor levels. Thus, changes in D2 receptor expression, binding, and signaling may be secondary to high-fat feeding that is coupled with weight gain and obesity.

Finally, the saturated HFD potentiated expression of the pGluR $1^{\text {Ser845 }}$ subunit of the AMPA receptor (AMPAR) in the NAc. AMPAR transmission in the NAc is a crucial control point for psychostimulant-related behaviors: phosphorylation at the Ser845 residue of GluR1 promotes AMPAR insertion, increases excitability, and is critical for synaptic plasticity (Loweth et al, 2014; van Huijstee and Mansvelder, 2014). It is not clear how such synaptic changes could contribute to suppressed AMPH responses by saturated dietary lipids. Palatable food and psychostimulants have been shown to increase pGluR1 ${ }^{\text {Ser845 }}$ in the NAc (Peng et al, 2011; Rauggi et al, 2005; Valjent et al, 2005), whereas increasing glutamatergic signaling in the NAc is reported to enhance goal-directed behavior for food (Stuber et al, 2011) and drug rewards (Cornish and Kalivas, 2000). We speculate that AMPAR-mediated synaptic adaptions by saturated HFD could underlie potential increases in reward-seeking behavior as a means to compensate for dampened mesolimbic function.

Future studies are warranted to identify how saturated lipid intake is modulating these NAc signaling elements; however, our data suggest that they do not stem from alterations in DA metabolism and biosynthesis given that NAc DA turnover and TH expression were similar across groups. However, interpretation of these tissue measures is limited by the fact that they do not provide a direct index of extracellular DA levels. Nonetheless, our findings are in agreement with data showing that 6 weeks of HFD fails to alter evoked DA release in the NAc of nonobese rats (Cone et $a l, 2013)$. On the other hand, there is evidence of decreased NAc DA flux in rats receiving HFD for 12 weeks (Davis et al, 2008). In view of the different diet durations used in these studies, it may be that longer periods ( $>8$ weeks) of high-fat feeding are required for decreases in NAc DA turnover in rats. Further studies are required to determine whether altered DA and glutamatergic signaling and changes in the conditioned behavioral responses to AMPH by saturated $\mathrm{HFD}$ are tied to alterations in in vivo DA release.

\section{SUMMARY}

Humans and animal studies have demonstrated associations between obesity and impaired reward function, but it has not been clear whether they are a consequence of the obese state, to what degree they depend on dietary components, and whether metabolic changes are involved. Although genetic components underlying obesity can affect DA signaling and modulate reward sensitivity in humans (Stice and Dagher, 2010; Stice et al, 2008) and rodents (Fulton et al, 2006; Geiger et al, 2009; Shin et al, 2011), several lines of evidence suggest secondary influence of the obese state on reward functions (Davis et al, 2008; Fulton et al, 2006; Geiger et al, 2009;
Johnson and Kenny, 2010; Shin et al, 2011). Our results show that a diet rich in saturated fat independent of weight gain and related metabolic changes impairs sensitivity to the rewarding and locomotor effects of AMPH and elicits neuroadaptations in the NAc coincident with reduced D1R signaling. In line with the reward deficiency hypothesis (Davis et al, 2004; Wang et al, 2002), could excess saturated fat intake contribute to neurobehavioral adaptations that promote increased consumption of rewarding, palatable foods as a means to compensate for reward hyposensitivity? This possibility is supported by findings showing that blunted AMPH responses are linked to increased risk for compulsive drug use in humans (Casey et al, 2014). Furthermore, anhedonia (reduced ability to experience pleasure from reward), a feature of depression, is exhibited by rodents consuming a saturated HFD (Sharma et al, 2013; Sharma and Fulton, 2013) and is associated with obesity in women (Davis et al, 2004). Indeed, the association between depression and obesity may be partly rooted in diets biased toward saturated fat intake (Hryhorczuk et al, 2013), as circulating levels of the saturated fatty acid palmitate correlate positively with depression severity in humans (Tsuboi et al, 2013). Determining the mechanisms by which overconsumption of saturated dietary lipids leads to perturbations in mesolimbic DA signaling may thus have important implications not only for our understanding of overeating and obesity but also for the link between mood disorders and metabolic disease.

\section{FUNDING AND DISCLOSURE}

This study was supported by a CIHR grant (MOP123280) and CIHR New Investigator salary award to SF, by a CIHR grant (MOP115042) and Fonds de Recherche Quebec-Santé salary award to TA, an NSERC (249848-2007 RGPIN) and FRQS group grant to the CSBN to AA, a CIHR grant (MOP9575) to CD, and doctoral scholarships from the Montreal Diabetes Research Center/Université de Montréal and CMDO/Novo Nordisk to $\mathrm{CH}$. The authors declare no conflict of interest.

\section{ACKNOWLEDGMENTS}

We thank Daniel Palacios and Patrick Augustyniak for their technical help.

\section{REFERENCES}

Abizaid A, Liu ZW, Andrews ZB, Shanabrough M, Borok E, Elsworth JD et al (2006). Ghrelin modulates the activity and synaptic input organization of midbrain dopamine neurons while promoting appetite. J Clin Invest 116: 3229-3239.

Bale TL, Baram TZ, Brown AS, Goldstein JM, Insel TR, McCarthy MM et al (2010). Early life programming and neurodevelopmental disorders. Biol Psychiatry 68: 314-319.

Beninger RJ, Nakonechny PL, Savina I (2003). cAMP-dependent protein kinase and reward-related learning: intra-accumbens Rp-cAMPS blocks amphetamine-produced place conditioning in rats. Psychopharmacology 170: 23-32.

Benoit SC, Kemp CJ, Elias CF, Abplanalp W, Herman JP, Migrenne $S$ et al (2009). Palmitic acid mediates hypothalamic insulin resistance by altering PKC-theta subcellular localization in rodents. J Clin Invest 119: 2577-2589. 
Buettner R, Parhofer KG, Woenckhaus M, Wrede CE, Kunz-Schughart LA, Scholmerich J et al (2006). Defining highfat-diet rat models: metabolic and molecular effects of different fat types. J Mol Endocrinol 36: 485-501.

Cagniard B, Sotnikova TD, Gainetdinov RR, Zhuang X (2014). The dopamine transporter expression level differentially affects responses to cocaine and amphetamine. J Neurogenet 28: 112-121.

Carr KD (2007). Chronic food restriction: enhancing effects on drug reward and striatal cell signaling. Physiol Behav 91: 459-472.

Carr KD, Kim G, Cabeza de Vaca S (2000). Hypoinsulinemia may mediate the lowering of self-stimulation thresholds by food restriction and streptozotocin-induced diabetes. Brain Res 863: 160-168.

Carsia RV, Weber H, McIlroy PJ, Hock CE (2008). Long-term dietary lipid regimen alters adrenocortical function at the cellular level. Horm Metab Rese 40: 848-853.

Casey KF, Benkelfat C, Cherkasova MV, Baker GB, Dagher A, Leyton M (2014). Reduced dopamine response to amphetamine in subjects at ultra-high risk for addiction. Biol Psychiatry 76: 23-30.

Cone JJ, Chartoff EH, Potter DN, Ebner SR, Roitman MF (2013). Prolonged high fat diet reduces dopamine reuptake without altering DAT gene expression. PLoS One 8: e58251.

Cornish JL, Kalivas PW (2000). Glutamate transmission in the nucleus accumbens mediates relapse in cocaine addiction. J Neurosci 20: Rc89.

Davidson TL, Monnot A, Neal AU, Martin AA, Horton JJ, Zheng W (2012). The effects of a high-energy diet on hippocampaldependent discrimination performance and blood-brain barrier integrity differ for diet-induced obese and diet-resistant rats. Physiol Behav 107: 26-33.

Davis C, Strachan S, Berkson M (2004). Sensitivity to reward: implications for overeating and overweight. Appetite 42: 131-138.

Davis JF, Tracy AL, Schurdak JD, Tschop MH, Lipton JW, Clegg DJ et al (2008). Exposure to elevated levels of dietary fat attenuates psychostimulant reward and mesolimbic dopamine turnover in the rat. Behav Neurosci 122: 1257-1263.

de Wit N, Derrien M, Bosch-Vermeulen H, Oosterink E, Keshtkar S, Duval C et al (2012). Saturated fat stimulates obesity and hepatic steatosis and affects gut microbiota composition by an enhanced overflow of dietary fat to the distal intestine. Am J Physiol Gastrointest Liver Physiol 303: G589-G599.

Fulton S, Pissios P, Manchon RP, Stiles L, Frank L, Pothos EN et al (2006). Leptin regulation of the mesoaccumbens dopamine pathway. Neuron 51: 811-822.

Fulton S, Woodside B, Shizgal P (2000). Modulation of brain reward circuitry by leptin. Science 287: 125-128.

Geiger BM, Haburcak M, Avena NM, Moyer MC, Hoebel BG, Pothos EN (2009). Deficits of mesolimbic dopamine neurotransmission in rat dietary obesity. Neuroscience 159: 1193-1199.

Gelinas R, Thompson-Legault J, Bouchard B, Daneault C, Mansour A, Gillis MA et al (2011). Prolonged QT interval and lipid alterations beyond beta-oxidation in very long-chain acylCoA dehydrogenase null mouse hearts. Am J Physiol Heart Circ Physiol 301: H813-H823.

Gerdjikov TV, Giles AC, Swain SN, Beninger RJ (2007). Nucleus accumbens PKA inhibition blocks acquisition but enhances expression of amphetamine-produced conditioned activity in rats. Psychopharmacology 190: 65-72.

Hamdi A, Porter J, Prasad C (1992). Decreased striatal D2 dopamine receptors in obese Zucker rats: changes during aging. Brain Res 589: 338-340.

Hernandez G, Haines E, Rajabi H, Stewart J, Arvanitogiannis A, Shizgal P (2007). Predictable and unpredictable rewards produce similar changes in dopamine tone. Behav Neurosci 121: 887-895.

Hiroi N, White NM (1991). The amphetamine conditioned place preference: differential involvement of dopamine receptor subtypes and two dopaminergic terminal areas. Brain Res 552: 141-152.
Hoefel AL, Hansen F, Rosa PD, Assis AM, Silveira SL, Denardin CC et al (2011). The effects of hypercaloric diets on glucose homeostasis in the rat: influence of saturated and monounsaturated dietary lipids. Cell Biochem Funct 29: 569-576.

Hryhorczuk C, Sharma S, Fulton SE (2013). Metabolic disturbances connecting obesity and depression. Front Neurosci 7: 177.

Johnson PM, Kenny PJ (2010). Dopamine D2 receptors in addiction-like reward dysfunction and compulsive eating in obese rats. Nat Neurosci 13: 635-641.

Jones SR, Gainetdinov RR, Wightman RM, Caron MG (1998). Mechanisms of amphetamine action revealed in mice lacking the dopamine transporter. J Neurosci 18: 1979-1986.

Kanoski SE, Zhang Y, Zheng W, Davidson TL (2010). The effects of a high-energy diet on hippocampal function and blood-brain barrier integrity in the rat. J Alzheimers Dis 21: 207-219.

Keita H, Ramirez-San Juan E, Paniagua-Castro N, GardunoSiciliano L, Quevedo L (2013). The long-term ingestion of a diet high in extra virgin olive oil produces obesity and insulin resistance but protects endothelial function in rats: a preliminary study. Diabetol Metab Syndr 5: 53.

Kien CL, Bunn JY, Stevens R, Bain J, Ikayeva O, Crain $\mathrm{K}$ et al (2014). Dietary intake of palmitate and oleate has broad impact on systemic and tissue lipid profiles in humans. Am J Clin Nutr 99: 436-445.

Kleinridders A, Schenten D, Konner AC, Belgardt BF, Mauer J, Okamura T et al (2009). MyD88 signaling in the CNS is required for development of fatty acid-induced leptin resistance and dietinduced obesity. Cell Metab 10: 249-259.

Labouebe G, Liu S, Dias C, Zou H, Wong JC, Karunakaran S et al (2013). Insulin induces long-term depression of ventral tegmental area dopamine neurons via endocannabinoids. Nat Neurosci 16: 300-308.

Lai JS, Hiles S, Bisquera A, Hure AJ, McEvoy M, Attia J (2014). A systematic review and meta-analysis of dietary patterns and depression in community-dwelling adults. Am J Clin Nutr 99: 181-197.

Loweth JA, Tseng KY, Wolf ME (2014). Adaptations in AMPA receptor transmission in the nucleus accumbens contributing to incubation of cocaine craving. Neuropharmacology 76 Pt B: 287-300.

Maric T, Woodside B, Luheshi GN (2014). The effects of dietary saturated fat on basal hypothalamic neuroinflammation in rats. Brain Behav Immun 36: 35-45.

Martinez-Gonzalez MA, Martin-Calvo N (2013). The major European dietary patterns and metabolic syndrome. Rev Endocr Metab Disord 14: 265-271.

Matyash V, Liebisch G, Kurzchalia TV, Shevchenko A, Schwudke D (2008). Lipid extraction by methyl-tert-butyl ether for highthroughput lipidomics. J Lipid Res 49: 1137-1146.

Morales L, Del Olmo N, Valladolid-Acebes I, Fole A, Cano V, Merino B et al (2012). Shift of circadian feeding pattern by highfat diets is coincident with reward deficits in obese mice. PLoS One 7: e36139.

Munzberg H, Flier JS, Bjorbaek C (2004). Region-specific leptin resistance within the hypothalamus of diet-induced obese mice. Endocrinology 145: 4880-4889.

Panchal SK, Poudyal H, Iyer A, Nazer R, Alam MA, Diwan V et al (2011). High-carbohydrate, high-fat diet-induced metabolic syndrome and cardiovascular remodeling in rats. J Cardiovasc Pharmacol 57: 611-624.

Peng XX, Ziff EB, Carr KD (2011). Effects of food restriction and sucrose intake on synaptic delivery of AMPA receptors in nucleus accumbens. Synapse (New York, NY) 65: 1024-1031.

Rauggi R, Scheggi S, Cassanelli A, De Montis MG, Tagliamonte A, Gambarana C (2005). The mesolimbic dopaminergic response to novel palatable food consumption increases dopamine-D1 receptor-mediated signalling with complex modifications of the DARPP-32 phosphorylation pattern. J Neurochem 92 : 867-877. 
Robinson TE, Becker JB (1986). Enduring changes in brain and behavior produced by chronic amphetamine administration: a review and evaluation of animal models of amphetamine psychosis. Brain Res 396: 157-198.

Rosqvist F, Iggman D, Kullberg J, Cedernaes J, Johansson HE, Larsson A et al (2014). Overfeeding polyunsaturated and saturated fat causes distinct effects on liver and visceral fat accumulation in humans. Diabetes 63: 2356-2368.

Sampey BP, Vanhoose AM, Winfield HM, Freemerman AJ, Muehlbauer MJ, Fueger PT et al (2011). Cafeteria diet is a robust model of human metabolic syndrome with liver and adipose inflammation: comparison to high-fat diet. Obesity (Silver Spring) 19: 1109-1117.

Sharma S, Fernandes MF, Fulton S (2013). Adaptations in brain reward circuitry underlie palatable food cravings and anxiety induced by high-fat diet withdrawal. Int J Obes 37: 1183-1191.

Sharma S, Fulton S (2013). Diet-induced obesity promotes depressive-like behaviour that is associated with neural adaptations in brain reward circuitry. Int J Obes 37: 382-389.

Shin AC, Townsend RL, Patterson LM, Berthoud HR (2011). "Liking" and "wanting" of sweet and oily food stimuli as affected by high-fat diet-induced obesity, weight loss, leptin, and genetic predisposition. Am J Physiol Regul Integr Comp Physiol 301: R1267-R1280.

Siciliano CA, Calipari ES, Jones SR (2014). Amphetamine potency varies with dopamine uptake rate across striatal subregions. J Neurochem 131: 348-355.

Spielewoy C, Biala G, Roubert C, Hamon M, Betancur C, Giros B (2001). Hypolocomotor effects of acute and daily d-amphetamine in mice lacking the dopamine transporter. Psychopharmacology 159: 2-9.

Stice E, Dagher A (2010). Genetic variation in dopaminergic reward in humans. Forum Nutr 63: 176-185.

Stice E, Spoor S, Bohon C, Small DM (2008). Relation between obesity and blunted striatal response to food is moderated by TaqIA A1 allele. Science 322: 449-452.

Stuber GD, Sparta DR, Stamatakis AM, van Leeuwen WA, Hardjoprajitno JE, Cho $S$ et al (2011). Excitatory transmission from the amygdala to nucleus accumbens facilitates reward seeking. Nature 475: 377-380.

Sulzer D, Chen TK, Lau YY, Kristensen H, Rayport S, Ewing A (1995). Amphetamine redistributes dopamine from synaptic vesicles to the cytosol and promotes reverse transport. J Neurosci 15: 4102-4108.

Taib B, Bouyakdan K, Hryhorczuk C, Rodaros D, Fulton S, Alquier T (2013). Glucose regulates hypothalamic long-chain fatty acid metabolism via AMP-activated kinase (AMPK) in neurons and astrocytes. J Biol Chem 288: 37216-37229.

Teegarden SL, Scott AN, Bale TL (2009). Early life exposure to a high fat diet promotes long-term changes in dietary preferences and central reward signaling. Neuroscience 162: 924-932.

Tellez LA, Medina S, Han W, Ferreira JG, Licona-Limon P, Ren X et al (2013). A gut lipid messenger links excess dietary fat to dopamine deficiency. Science 341: 800-802.
Thaler JP, Yi CX, Schur EA, Guyenet SJ, Hwang BH, Dietrich MO et al (2012). Obesity is associated with hypothalamic injury in rodents and humans. J Clin Invest 122: 153-162.

Thanos PK, Kim R, Cho J, Michaelides M, Anderson BJ, Primeaux $\mathrm{SD}$ et al (2010). Obesity-resistant S5B rats showed greater cocaine conditioned place preference than the obesity-prone $\mathrm{OM}$ rats. Physiol Behav 101: 713-718.

Tsuboi H, Watanabe M, Kobayashi F, Kimura K, Kinae N (2013). Associations of depressive symptoms with serum proportions of palmitic and arachidonic acids, and alpha-tocopherol effects among male population-a preliminary study. Clin Nutr 32: 289-293.

Valdearcos M, Robblee MM, Benjamin DI, Nomura DK, Xu AW, Koliwad SK (2014). Microglia dictate the impact of saturated fat consumption on hypothalamic inflammation and neuronal function. Cell Rep 9: 2124-2138.

Valjent E, Pascoli V, Svenningsson P, Paul S, Enslen H, Corvol JC et al (2005). Regulation of a protein phosphatase cascade allows convergent dopamine and glutamate signals to activate ERK in the striatum. Proc Natl Acad Sci USA 102: 491-496.

van Dijk SJ, Feskens EJ, Bos MB, Hoelen DW, Heijligenberg R, Bromhaar MG et al (2009). A saturated fatty acid-rich diet induces an obesity-linked proinflammatory gene expression profile in adipose tissue of subjects at risk of metabolic syndrome. Am J Clin Nutr 90: 1656-1664.

van Huijstee AN, Mansvelder HD (2014). Glutamatergic synaptic plasticity in the mesocorticolimbic system in addiction. Front Cell Neurosci 8: 466.

Vezina P (2004). Sensitization of midbrain dopamine neuron reactivity and the self-administration of psychomotor stimulant drugs. Neurosci Biobehav Rev 27: 827-839.

Volkow ND, Wang GJ, Telang F, Fowler JS, Thanos PK, Logan J et al (2008). Low dopamine striatal D2 receptors are associated with prefrontal metabolism in obese subjects: possible contributing factors. Neuroimage 42: 1537-1543.

Vucetic Z, Carlin JL, Totoki K, Reyes TM (2012). Epigenetic dysregulation of the dopamine system in diet-induced obesity. J Neurochem 120: 891-898.

Wang GJ, Volkow ND, Fowler JS (2002). The role of dopamine in motivation for food in humans: implications for obesity. Exp Opin Ther Targets 6: 601-609.

Wang GJ, Volkow ND, Logan J, Pappas NR, Wong CT, Zhu W et al (2001a). Brain dopamine and obesity. Lancet 357: 354-357.

Wang J, Obici S, Morgan K, Barzilai N, Feng Z, Rossetti L (2001b). Overfeeding rapidly induces leptin and insulin resistance. Diabetes 50: 2786-2791.

Wang JQ, McGinty JF (1997). The full D1 dopamine receptor agonist SKF-82958 induces neuropeptide mRNA in the normosensitive striatum of rats: regulation of D1/D2 interactions by muscarinic receptors. J Pharmacol Exp Ther 281: 972-982.

Wolf ME (1998). The role of excitatory amino acids in behavioral sensitization to psychomotor stimulants. Prog Neurobiol 54: $679-720$.

Supplementary Information accompanies the paper on the Neuropsychopharmacology website (http://www.nature.com/npp) 